\title{
Giant Plexiform Neurofibroma with Hemorrhage in Cranio-maxillofacial Region as Depicted on CT AND MRI
}

\author{
Y. Feng, Z. Yang, T. Chen, Q.,Wang, W. Deng
}

Department of Radiology, West China Hospital of Sichuan University, Chengdu, Sichuan, China

\begin{abstract}
Plexiform neurofibroma $(\mathrm{PN})$ is a rare benign tumor and a special subtype of neurofibromatosis type 1 (NF1). Though the incidence is low, giant PN of the craniomaxillofacial region could result in severe hemifacial hypertrophy which is known as a typical manifestation of NF1 in young children. Here, we retrospectively reported a giant plexiform neurofibroma with hemorrhage in the cranio-maxillofacial region detected by CT and MRI. In addition, a brief review of the relevant literature is presented.
\end{abstract}

Key words: Plexiform neurofibroma, Hemorrhage, CT, MR

\section{INTRODUCTION}

Neurofibromatosis is an autosomal dominant phakomatoses or neurocutaneous syndrome. Two distinct types of neurofibromatosis including neurofibromatosis type 1 (NF1) and neurofibromatosis type 2 (NF2) have been recognized according to the different clinical manifestations and genetic mutation. Plexiform neurofibroma $(\mathrm{PN})$, a special subtype of $\mathrm{NF} 1$, is a rare, slow-growing benign tumor [1]. Although previous study presented one case with PN in the craniomaxillofacial region, the entity with hemorrhage in this region on CT and MRI has not been described [2]. We report here the radiological findings of a giant hemorraghic $\mathrm{PN}$ in the cranio-maxillofacial region to illustrate the usefulness of CT or MRI in making diagnosis.

\section{CASE REPORT}

A 21-year-old man presented with a mass on the right side of his face for 16 years, which had initially appeared as a small cutaneous protuberance with a size of $2 \mathrm{~cm}$ in diameter in the right zygomatic arch region. The mass gradually enlarged and involved the right scalp, eye and face, and the color turned darker and darker with time. No treatments were administered in the history. Nine days before admission, the lesion grew abruptly, which resulted in cosmetic impairment of right face accompanied by severe headache, and the proptosis of the right eye and loss of eyesight. No other first-degree relatives in his family were noted NF1 besides his grandmother with neurofibroma alongside of left eye.
Nine days after abrupt enlargement, the patient was admitted for treatment, and emergent CT was performed. The next day after the admission, the patient underwent enhanced CT and MRI scans of the craniomaxillofacial region. The studies revealed a giant cystic and solid mass with the size of $30 \mathrm{~cm} \times 25 \mathrm{~cm} \times 16 \mathrm{~cm}$, involving the right eye, right auricle, and subcutaneous and deep soft tissues in the face. The boundary of the mass was not clear and invaded the surrounding structures. The cyst within the mass was filled predominantly with subacute hemorrhage appearing as hyper- and iso- attenuation on plain CT scan (Fig. 1A) and non-enhanced on contrast-enhanced CT image (Fig. 1B), heterogeneously low to high signal intensity on T1-weighted image, and intermediate to light high signal intensity on T2-weighted image (Fig. 1D-E). The small amount of solid components presented with heterogeneously intermediate to low signal intensity on non-enhanced T1-weighted image and intermediate to high signal intensity on T2-weighted image, and light enhancement on contrasted-enhanced T1-weighted image. Heterogeneous enhancement and small abnormal vessels were seen within the solid lesion of the mass on contrast-enhanced CT and MRI, whereas no enhancement was found in the cyst. Bony defects were found within the cavalla, temporal bone and top of fossa orbitalis on CT and MRI, and the adjacent right skull base was thickened and sclerotic on CT. The dysplastic right temporal lobe complicated with a meningoencephalocele was noted, and the right frontal lobe was atrophic. The right fossa orbitalis was enlarged and the right maxilla was also dysplastic. The optic nerve and extraocular muscles were involved by the mass, The CT surface shaded display (SSD) reconstruction showed the cosmetic impairment of right face proptosis of the right eye and the right ear was lower than normal in location(Fig. 1C).

Subsequently, the patient underwent surgery, and venous sinusoids and hematoma were found within the mass with defective temporal bones. The lesion was adherent to both the intracranial and craniofacial region. The right fossa orbitalis, orbital plate, and lateral rectus muscle were involved, and the zygomatic arch and articulatio mandibularis were deformed. The solid part of tumor is consisted of spindle-like schwann cells, and the plexiform neurofibroma was pathologically confirmed after surgical procedure (Fig. 2A-B). Owing to the resection of the tumor, facial ptosis strikingly improved one month later, and the patient was then recovered step by step. 

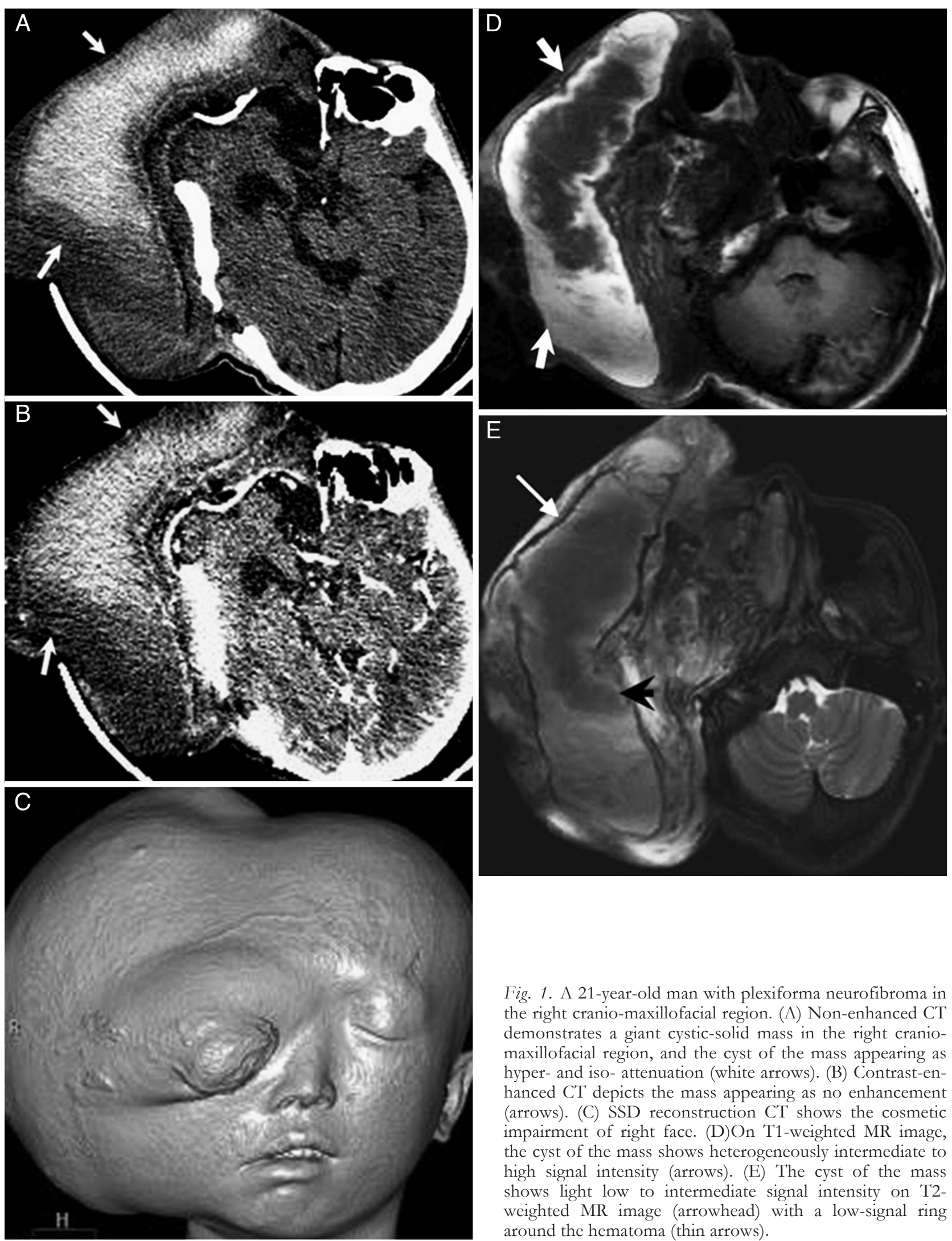

Fig. 1. A 21-year-old man with plexiforma neurofibroma in the right cranio-maxillofacial region. (A) Non-enhanced CT demonstrates a giant cystic-solid mass in the right craniomaxillofacial region, and the cyst of the mass appearing as hyper- and iso- attenuation (white arrows). (B) Contrast-enhanced CT depicts the mass appearing as no enhancement (arrows). (C) SSD reconstruction CT shows the cosmetic impairment of right face. (D)On T1-weighted MR image, the cyst of the mass shows heterogeneously intermediate to high signal intensity (arrows). (E) The cyst of the mass shows light low to intermediate signal intensity on T2weighted MR image (arrowhead) with a low-signal ring around the hematoma (thin arrows).

\section{Discussion}

NF1 is the most common type of neurofibromatosis and usually presented with involvement of bone, nervous system, soft tissues and skin [3]. The mutated gene for NF1 has been located to chromosome
$17 q 11.2$ [4]. PN is a special subtype of NF1 usually presented in childhood and 50\% individuals have a positive family history as in the present case. Malformation is a common feature due to invasive growth of the tumor, with hyper-pigmentation of the overlying involved skin. In the present case, PN which arises 

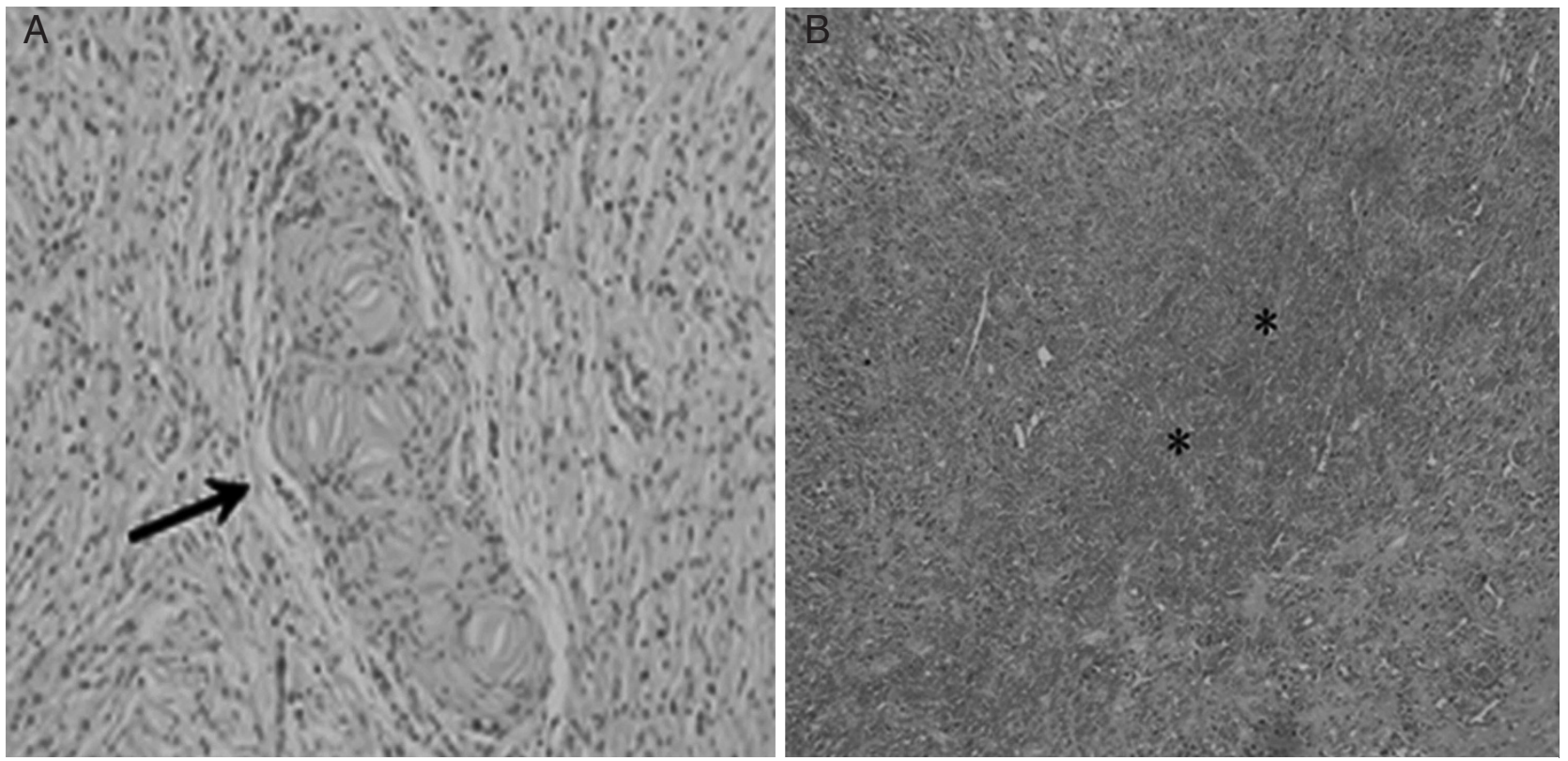

Fig. 2. Photomicrography of the case mention in figure 1. (A) Histology specimen demonstrates that the tumor is consisted of spindle-like schwann cells (arrow) (HE $200 \times$ ), and (B) the area of hemorrhage in the mass (*, HE $100 \times$ ).

from cranio-maxillofacial region may involve the five sensory organs malformation is observed as well as a dysplastic cranium. The rapid increase of the PN size due to subacute hemorrhage within the tumor caused the loss of eyesight is extremely rare in our report [5].

PN arises mainly from the peripheral nerves and represents a neoplasia of the nerve trunk or peripheral nerves and their nerve endings. The tumor consists of neuronal axons, Schwann cells, fibroblasts and peripheral nerves with mucinous degeneration are predominant manifestations under electron microscope. Hyperplasia of Schwann cells is more common with the typical network-like growth [6]. PN consists of a proliferation of cells in the nerve sheath extending across the length of a nerve without a capsule and involving adjacent tissues [7]. Treatment of giant $\mathrm{PN}$ is typically surgical and complete resection is often difficult due to the extensive growth of the tumor and residual apartment may develop malignant change [8].

Non-enhanced CT scan demonstrated a giant heterogeneous attenuation mass with extensive involvement of both the superficial and deep tissue within cranio-maxillofacial region. Hyper attenuation areas on CT were compatible with hemorrhage. MRI displayed a heterogeneously hypo-intense to iso-intense mass on T1-weighted MRI which was hyper-intense on T2-weighted images compatible with a cyst-like appearance with subacute hemorrhage and mucinous degeneration. Heterogeneous enhancement suggests multiple components along with abnormal vessels involving the neurovascular bundle $[9,10]$.

Both CT and MRI can disclose the internal features of the tumor and the invasion of the surrounding tissues. In addition, the entire lesion can be clearly displayed in multiple planes using MRI and three-dimensional CT. SSD can reconstruct the profile of PN. MRI can effectively disclose the composition of the various soft tissues and the components of subacute hematoma such as hemosiderin. T2-weighted MRI has an advantage in differential diagnosis over CT [11].

In summary, we report a case of giant PN with hemorrhage in right cranio-maxillofacial region and its appearances on CT and MRI. The images can display the internal features of the mass, and relationship between the three-dimensional structure of the lesion and the neighboring structures. All findings could shed a light on the future surgical resection.

\section{REFERENCES}

1. Overdiek A, Feifel H, Schaper J, Mayatepek E, Rosenbaum T. Diagnostic delay of NF1 in hemifacial hypertrophy due to plexiform neurofibromas. No To Hattasu (Brain and Development). 2006 Feb 14;28(5):275-280.

2. Wise JB, Patel SG, Shah JP. Management issues in massive pediatric facial plexiform neurofibroma with neurofibromatosis type 1. Head Neck. 2002 Feb 2; 24: 207-211.

3. Hersh JH. Health supervision for children with neurofibromatosis. Pediatrics 2008 March; 21: 633-642.

4. Cre'ange A, Zeller J, Rostaing-Rigattieri S, Brugie 'res P, Degos JD, Revuz J, et al. Neurological complications of neurofibromatosis type 1 in adulthood. Brain. 1999 Feb 27; 122: 473-481.

5. Malagari K, Drakopoulos S, Brountzos E, Sissopulos A, Efthimidadou A, Hadjiyiannakis E, et al. Plexiform neurofibroma of the liver: findings on MR imaging, angiography, and CT portography. AJR. 2001 Jan 31; 176:493-495.

6. Waggoner DJ, Towbin J, Gottesman G, Gutmann DH. Clinic-based study of plexiform neurofibromas in neurofibromatosis I. Am J Med Genet. 2001 Feb; 9:132-135.

7. Aribandi M, Wood WE, Elston, DM, Weiss DL. CT features of plexiform neurofibroma of the submandibular gland. AJNR. 2006 Jan; 27:126-128.

8. Valeyrie-Allanore L, Ismai"li, Bastuji-Garin N, Zeller J, Wechsler J, Revuz J, et al. Symptoms associated with malignancy of peripheral nerve sheath tumours: a retrospective study of 69 patients with neurofibromatosis $1 . \mathrm{Br} J$ Dermatol. 2005 Jun 27;153: 79-82.

9. Lin J, Martel W. Cross-sectional imaging of peripheral 
nerve sheath tumors: characteristic signs on CT, MR imaging, and sonography. AJR. 2001 Jan; 176:75-82.

10. Bourgeois JM, Radhi J, Elden L, et al. Plexiform neurofibroma of the submandibular salivary gland in a child. Can J Gastroenterol. 2001 Dec; 15:835-37.

11. Fortman BJ, Kuszyk BS, Urban B A. Fishman EK. Neurofibromatosis type 1: a diagnostic mimicker at CT. Radiographics. 2001 May; 21:601-612. Review.
Received: September 9, 2009 / Accepted: January 5, 2010

Address for correspondence:

Zhi-gang Yang

Department of Radiology

West China Hospital of Sichuan University

37\# Guo Xue Xiang

Chengdu, Sichuan 610041

China

Tel/Fax: +86-28/85423817(O)

E-mail: yangzg1117@yahoo.com.cn 